\title{
Cumulative Burden of Glucocorticoid-related Adverse Events in Patients with Systemic Lupus Erythematosus: Findings from a 12-year Longitudinal Study
}

\author{
Hung-Lin Chen, Li-Jiuan Shen, Ping-Ning Hsu, Chieh-Yu Shen, Susan A. Hall, \\ and Fei-Yuan Hsiao
}

ABSTRACT. Objective. The aim of this population-based study is to examine the adverse events (AE) associated with longitudinal systemic glucocorticoid (GC) use among an ethnic Chinese systemic lupus erythematosus (SLE) cohort.

Methods. Our study subjects were patients with newly diagnosed SLE aged 18 and older who received at least 1 prescription of systemic GC between 2001 and 2012 from Taiwan's National Health Insurance Research Database (NHIRD). The earliest prescription date of systemic GC for each subject was defined as the index date. For each subject, we calculated the average prednisolone-equivalent dose and the medication possession ratio (MPR) of GC use every 90 days for each patient after the index date. Patients with a diagnosis of AE (defined by the International Classification of Diseases-9-Clinical Modification diagnosis code) during the followup were also identified from the NHIRD. Generalized estimating equations adjusted for propensity score were applied to examine the association between longitudinal GC use and risks of prespecified AE (musculoskeletal, gastrointestinal, ophthalmologic, infectious, cardiovascular, neuropsychiatric, metabolic, and dermatologic diseases).

Results. We identified 11,288 patients with SLE (mean followup: $6.28 \mathrm{yrs}$ ). Higher doses and higher MPR of GC were associated with increased risk of osteonecrosis [adjusted OR (aOR) 2.87-9.09]. Similar results were found regarding the risk of osteoporosis (aOR 1.71-3.67), bacterial infection (aOR 2.12-3.89), Cushingoid syndrome (aOR 6.51-62.03), and sleep disorder (aOR 1.42-3.59).

Conclusion. To our knowledge, this is the first study to show that the dose and intensity of longitudinal use of GC were both associated with risk of AE among a nationwide Asian SLE cohort. (First Release November 15 2017; J Rheumatol 2018;45:83-9; doi:10.3899/jrheum.160214)

Key Indexing Terms:

SYSTEMIC LUPUS ERYTHEMATOSUS GLUCOCORTICOIDS ADVERSE EVENTS NATIONAL HEALTH INSURANCE RESEARCH DATABASE

\footnotetext{
From the Graduate Institute of Clinical Pharmacy, the Graduate Institute of Immunology, the Graduate Institute of Clinical Medicine, and the School of Pharmacy, College of Medicine, National Taiwan University; Department of Internal Medicine, and Department of Pharmacy, National Taiwan University Hospital, Taipei, Taiwan; Department of Epidemiology, Biogen, Cambridge, Massachusetts, USA.

Biogen Inc. provided funding for this project.

H.L. Chen, MS, Research Associate, Graduate Institute of Clinical Pharmacy, College of Medicine, National Taiwan University; L.J. Shen, PhD, Associate Professor, Graduate Institute of Clinical Pharmacy, and School of Pharmacy, College of Medicine, National Taiwan University, and Director, Department of Pharmacy, National Taiwan University Hospital; P.N. Hsu, MD, PhD, Attending Physician, Department of Internal Medicine, National Taiwan University Hospital, and Professor, Graduate Institute of Immunology, College of Medicine, National Taiwan University; C.Y. Shen, MD, Visiting Physician, Department of Internal Medicine, National Taiwan University Hospital, and Doctoral Student, Graduate Institute of Clinical Medicine, College of Medicine, National Taiwan University; S.A. Hall, PhD, Associate Director, Department of Epidemiology, Biogen; F.Y. Hsiao, PhD, Associate Professor, Graduate Institute of Clinical Pharmacy, and School of Pharmacy, College of Medicine, National Taiwan University, and Adjunct Associate Researcher, Department of Pharmacy, National Taiwan University Hospital. H.L. Chen and L.J. Shen contributed equally as first authors.

Address correspondence to F.Y. Hsiao, National Taiwan University,

College of Medicine, Graduate Institute of Clinical Pharmacy, Room 220,

33 Linsen South Road, Taipei 10050,Taiwan.E-mail: fyshsiao@ntu.edu.tw

Accepted for publication July 31, 2017.
}

Systemic lupus erythematosus (SLE) is a complicated chronic inflammatory autoimmune disease of unclear etiology, affecting 124 self-report cases per 1000 persons $^{1}$. Over the past few decades, owing to improvements in diagnostics and treatment, the overall 5-year survival rate of patients with SLE has significantly improved from $75 \%$ in the 1950 s to $95 \%$ in $2010^{2}$. As the life expectancy of patients with SLE has been prolonged, damage accrual due to disease flares and cumulative treatments has been suggested to be associated with a higher risk of mortality after 10 years of followup in this patient population ${ }^{3,4}$. Because there is no curative treatment for SLE, systemic glucocorticoids (GC) are the cornerstone of management that aims to halt acute disease progression, sometimes used in combination with other "steroid-sparing" agents to postpone the timing of the next flare ${ }^{5,6}$.

Despite the clinical efficacy of the antiinflammatory and immunosuppressive properties of GC in treating SLE, numerous adverse events (AE) have been observed in GC users. A retrospective cohort study found that patients with SLE receiving steroids were $1.5 \times$ more likely to develop AE, including chronic AE such as hypertension (HTN), cataracts,

Personal non-commercial use only. The Journal of Rheumatology Copyright @ 2018 . All rights reserved. 
migraines, and sleep disturbances, and acute AE such as fungal infections, herpes zoster, nausea/vomiting, and pneumonia ${ }^{7}$. High dose of steroids was particularly reported to be associated with higher risk of AE. For example, Ruiz-Arruza, et al reported that patients taking medium-high doses of prednisone had a higher risk of accruing damage than those taking no prednisone ${ }^{8}$.

Petri, et al also reported that the estimated rates of $\mathrm{AE}$ (including osteoporosis, obesity, mood changes, diabetes, fractures, and avascular necrosis) were at least doubled in patients with SLE receiving prednisolone-equivalent doses $>20 \mathrm{mg}$ /day compared with those receiving $\leq 20 \mathrm{mg} /$ day $^{9}$. Other studies have also suggested that permanent organ damage in patients with SLE was associated with longterm GC use ${ }^{10,11}$. However, existing studies are all limited by small sample size ${ }^{8,12}$, cross-sectional study design, or short followup time ${ }^{13,14}$, and may only identify temporary associations between $\mathrm{GC}$ use and associated $\mathrm{AE}^{3,14,15}$. Little is known about the association between the risk of GC-related $\mathrm{AE}$ and longitudinal patterns of GC use, in which dosage is influenced by fluctuating disease activity, patient characteristics, and comorbidity status, as well as by other concurrently administered SLE treatments.

To address these limitations, we took advantage of Taiwan's National Health Insurance Research Database (NHIRD), a longitudinal nationwide claims database, to investigate the longitudinal (in particular, time-varying) GC use and the incidence of GC-related $\mathrm{AE}$ among patients with SLE during a 10-year followup period.

\section{MATERIALS AND METHODS}

Data source. The data source was the NHIRD, a nationwide database containing claims records from Taiwan's mandatory National Health Insurance program. The program was launched in 1995 and covers over $99 \%$ of the population in Taiwan (23.12 million in 2009). Demographic and healthcare use information, including outpatient visits, hospital admissions, and prescription medications of each beneficiary, is systematically recorded in the NHIRD. The database has been described in detail elsewhere and has become the source for numerous epidemiological studies published in peer-reviewed journals ${ }^{16}$. All claims data during the period of January 1 , 2000 , to December 31, 2012, were incorporated in our study.

Ethical statement. Because the identification numbers of all subjects in the NHIRD were encrypted to protect individual privacy, this study was exempted from full review by the Institutional Review Board of the National Taiwan University Hospital and informed consent was waived.

Study cohort. Our study cohort consisted of newly diagnosed SLE patients aged 18 and older between January 1, 2001, and December 31, 2012. Patients with SLE were defined as having at least 1 inpatient or outpatient visit with a diagnosis of SLE [International Classification of Diseases-9-Clinical Modification $(\text { ICD-9-CM) }=710.0 \mathrm{x}]^{17}$ in the NHIRD. These patients with SLE were further validated using the Catastrophic Illness Database (consisting of the records of patients diagnosed with cancer or autoimmune diseases, including SLE). All NHI beneficiaries diagnosed with SLE are eligible to apply for a certificate of catastrophic illness, to be exempted from all copayments. Because one is required to have an official certificate of SLE diagnosis from the hospital to be eligible for the certificate of catastrophic illness, we were able to precisely identify the SLE cases and to avoid potential misclassification through the Catastrophic Illness Dataset. This approach has also been adopted by several published studies using the NHIRD as the data source ${ }^{18,19}$. The date of the earliest diagnosis of SLE was defined as the cohort entry date of our study subject. A 12-month washout period before the cohort entry date was used to ensure that these patients were newly diagnosed with SLE. Patients who never used GC during the study period were further excluded, because they may represent a specific SLE patient population, such as those asymptomatic or with very mild symptoms, which could be very different from those receiving GC. The index date was the earliest date among the recorded dates of patients receiving systemic GC. The 12 months before the index date served as the baseline information-gathering period to identify each patient's comorbidities, including ischemic heart disease, cerebrovascular disease, dyslipidemia, chronic obstructive pulmonary disease, diabetes mellitus, and renal dysfunction.

GC and other SLE treatments. Information about GC use and other SLE treatments was retrieved from the NHIRD quarterly (90 days) after the index date. The GC included in our study were oral and parenteral forms of cortisone, hydrocortisone, prednisolone, triamcinolone, betamethasone, dexamethasone, methylprednisolone, and paramethasone.

We further categorized GC use during each observational unit (i.e., 90-day interval) of the followup period into 4 groups based on the dose and intensity of GC use. The average daily and cumulative doses of GC were converted into the prednisolone-equivalent daily dose (Supplementary Table 1 , available with the online version of this article $)^{5}$. The intensity of exposure to GC was calculated by the medication possession ratio (MPR) as the total prescribing days of GC divided by the total number of days of each observational period (a value ranging from 0 to $100 \%)^{20}$. For example, if a patient receives a total 72 days of GC in the 90-day observational unit (our study), then the MPR of GC use is $80 \%$. An MPR of $\geq 80 \%$ was defined as "high intensity" based on the consensus of existing studies ${ }^{20}$. The pattern of GC use was then categorized into 4 groups: $\leq 10 \mathrm{mg}$ prednisolone-equivalent daily dose + MPR $<80 \%$ (low dose + low intensity), $\leq 10 \mathrm{mg}$ prednisolone-equivalent daily dose + MPR $\geq 80 \%$ (low dose + high intensity), $>10$ mg prednisolone-equivalent daily dose + MPR $<80 \%$ (high dose + low intensity), and $>10 \mathrm{mg}$ prednisolone-equivalent daily dose $+\mathrm{MPR} \geq 80 \%$ (high dose + high intensity).

In addition to GC usage, information about usage of other SLE treatments was retrieved in each observational unit (i.e., 90 days) during the followup period for each patient with SLE. Those treatments included antimalarials [such as hydroxychloroquine (HCQ)], immunosuppressive agents [cyclophosphamide (CYC), azathioprine (AZA), mycophenolate mofetil], and nonsteroidal antiinflammatory drugs (NSAID).

Outcome measurement. SLE patients with a diagnosis of AE (defined by the ICD-9-CM diagnosis code) during the observational period (2000-2012) were identified from the NHIRD. Acute AE included in our study were fracture, peptic ulcer, viral hepatitis, pancreatitis, nausea/vomiting, gout/hyperuricemia, ischemic heart disease, tuberculosis, fungal infection, and bacterial infection. Chronic AE included in this study were osteoporosis, osteonecrosis, myopathy, fatty liver, cataracts, glaucoma, psychosis, depression, sleep disorder, dyslipidemia, cerebrovascular disease, HTN, atherosclerosis, heart failure, obesity, Cushingoid syndrome, and acne vulgaris (Supplementary Table 2, available with the online version of this article). In some instances, in which an $\mathrm{AE}$ occurred, treatment for the $\mathrm{AE}$ with a drug typically used to treat a common GC-related AE, such as an antibiotic, antiosteoporosis agent, antiulcer agent, or antiviral agent, was used as a surrogate to identify it as an occurrence of a GC-related AE. Only incident AE after GC use were included in our analyses. To identify incident AE in our study, a 3-month washout period before the index diagnosis date was used for "acute" AE (such as peptic ulcer or infection) and a 12-month washout period before the index diagnosis date was used for "chronic" AE (such as osteoporosis or sleep disorder). The incidence of GC-related AE (both acute and chronic), the primary outcome of interest, was calculated as the number of new cases of a specific AE divided by the followup time (per 1000 person-yrs) 
To ensure that the occurrence of an AE was indeed GC-related, patients were required to be free of the particular AE during a washout period before the index date. The length of the washout period was defined as 30 days for acute $\mathrm{AE}$ and 180 days for chronic AE, respectively.

Statistical analysis. Generalized estimating equation (GEE) models with an autoregressive (AR-1) correlation structure that accounted for the intraclass correlation between repeated measurements for the same subjects were used to examine the association between GC use and clinical outcomes (i.e., GC-related AE). The GEE models were adjusted for patient demographics (age, sex), comorbidities, and concomitant SLE treatments. We also used a propensity score to address the potential selection bias among patients receiving high doses of GC. The propensity score was assigned based on the probability that an individual would receive a high dose of GC, and was estimated by a logistic regression model for observed covariates. Covariates included in the propensity score models were age, sex, comorbidities (including other autoimmune diseases), SLE severity index (patients were evaluated within 6-mos of cohort entry and categorized as having mild, moderate or severe SLE), and receipt of other SLE treatment ${ }^{21}$. We adopted a validated claims-based algorithm proposed by Garris, et al to estimate the SLE severity index in our study ${ }^{22}$. The severity of SLE of each patient was classified as "mild," "moderate," or "severe" based upon a combination of diagnosis codes, visits to rheumatologists, and filled prescriptions for SLE treatment (including corticosteroids, antimalarials, and immunosuppresives) in claims data. For example, a patient was classified as a "severe" SLE patient if he or she received oral corticosteroids with prednisone-equivalent dose $>40 \mathrm{mg} /$ day and CYC and was ever admitted to hospital with a primary diagnosis of SLE (ICD-9-CM codes 710) or SLE-related conditions ${ }^{22}$. The estimated propensity score of each patient was treated as a covariate and adjusted in the GEE models ${ }^{21}$. The associations between GC use and AE were presented as adjusted OR (aOR) with a 95\% CI. All comparisons and $\mathrm{aOR}$ were estimated using intervals with no exposure to $\mathrm{GC}$ as the reference group. The $\mathrm{p}$ value was 2 -sided, and $\alpha$ was set to 0.05 . All statistical analyses were performed using SAS (SAS 9.3; SAS Institute).

\section{RESULTS}

Patient characteristics. We identified 11,288 newly diagnosed SLE patients with a least 1 prescription of systemic GC (Figure 1). The mean age ( \pm SD) of the study cohort was 39.41 years ( $\pm 15.48 \mathrm{yrs}$ ). About $87 \%$ of our patients were women. Nearly one-third of these patients were in a severe disease stage based on the SLE severity index (mild: $36.4 \%$, moderate: $34.4 \%$, and severe: $29.2 \%$ ). Most patients with SLE in our study were taking GC (92.27\%), NSAID (79.32\%), and HCQ $(79.71 \%)$ at 1 year after cohort entry. These drugs are recommended for treating mild to moderate disease in clinical practice. Immunosuppressants such as AZA (31.31\%) or CYC (15.12\%), usually reserved for severe, life-threatening, or organ-threatening disease, were less common at the first year (Table 1).

$G C$-related AE. Table 2 summarizes the time-to-event and the incidence rate of the GC-related AE studied. Peptic ulcer (6251 events; $147.84 / 1000$ person-yrs) was the most frequently identified GC-related AE in our study cohort, followed by bacterial infection (5489 events; $136.42 / 1000$ person-yrs), insomnia (4019 events; 78.64/1000 person-yrs), fungal infection (2568 events; 42.26/1000 person-yrs), and HTN (2301 events; 37.67/1000 person-yrs). Over 1000 events of each of the following AE were identified in our study cohort: gout and hyperuricemia, osteoporosis, dyslipi-
SLE patients identified using ICD-9-CM code [710.0x] from inpatient or outpatient claims, double confirmed by Catastrophic illness database and had records in NHIRD during 2000-2012 (n=22,737)
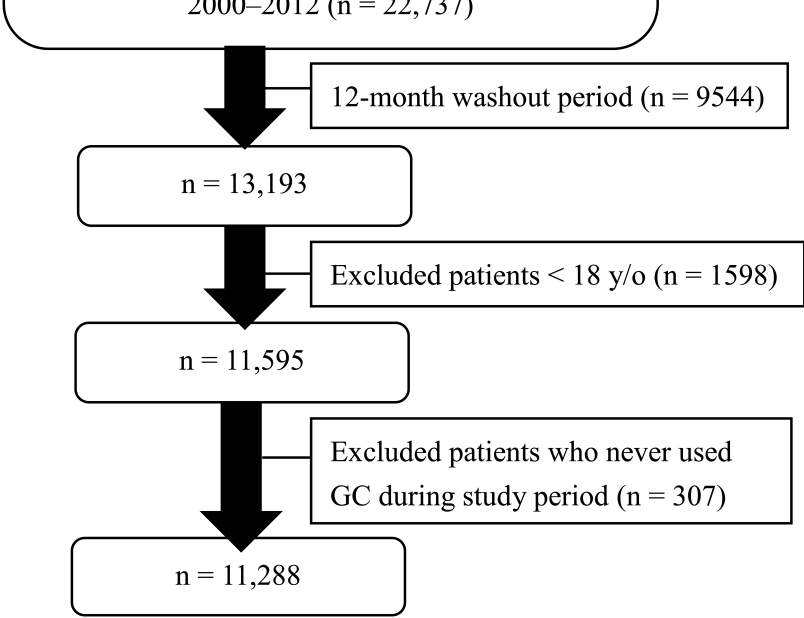

Figure 1. Flow chart showing the sampling procedure of the study cohort. SLE: systemic lupus erythematosus; ICD-9-CM: International Classification of Diseases-9-Clinical Modification; NHIRD: National Health Insurance Research Database; y/o: year-old; GC: glucocorticoid.

demia, cataract, fracture, nausea/vomiting, acne vulgaris, and depression (incidence ranged from 29.81 to $15.07 / 1000$ person-yrs).

Multivariate GEE models (Table 3) showed that high-dose and high-MPR GC use were associated with increased risk of osteonecrosis [aOR ranged from 2.87 to 9.09 ; $\leq 10 \mathrm{mg}+$ MPR < 80\%: aOR 2.87 (95\% CI 1.16-7.08); $\leq 10 \mathrm{mg}+$ MPR $\geq 80 \%: 5.04$ (2.14-11.91); $>10 \mathrm{mg}+$ MPR $<80 \%$ : 4.02 (1.70-9.49); $>10 \mathrm{mg}+\mathrm{MPR} \geq 80 \%$ (high dose + high intensity): 9.09 (3.70-22.33)]. Similar results were found regarding the risk of osteoporosis (aOR ranged from 1.71 to 3.67), bacterial infection (aOR ranged from 2.12 to 3.89), Cushingoid syndrome (aOR ranged from 6.51 to 62.03 ), and sleep disorder (aOR ranged from 1.42 to 3.59 ).

For peptic ulcer, viral hepatitis, cataracts, hyperuricemia, or acne vulgaris, our study showed that even patients exposed to high-intensity but low-dose GC therapy were at a higher risk of $\mathrm{AE}$ compared to those not exposed to $\mathrm{GC}$ [e.g., cataracts, $\leq 10 \mathrm{mg}+\mathrm{MPR}<80 \%: 1.28(0.92-1.77) ; \leq 10 \mathrm{mg}$ + MPR $\geq 80 \%$ : 1.74 (1.29-2.34); $>10 \mathrm{mg}+\mathrm{MPR}<80 \%$ : $1.68(1.22-2.31) ;>10 \mathrm{mg}+\mathrm{MPR} \geq 80 \%: 2.63(1.86-3.74)]$. For some types of AE, a higher risk was only seen in SLE patients with high-dose GC use, not those with low-dose GC use, such as myopathy [ $\leq 10 \mathrm{mg}+$ MPR $<80 \%$ : 0.91 $(0.08-10.31) ; \leq 10 \mathrm{mg}+\mathrm{MPR} \geq 80 \%$ : $0.71(0.05-9.98)$; $>10 \mathrm{mg}+$ MPR < 80\%: 8.92 (1.72-46.18); > $10 \mathrm{mg}+$ MPR $\geq 80 \%: 12.43$ (2.31-66.98)]. Such a pattern of association was also found regarding the risk of glaucoma,

Personal non-commercial use only. The Journal of Rheumatology Copyright $\odot$ 2018. All rights reserved. 
Table 1. Baseline characteristics of newly diagnosed SLE patients $(\mathrm{n}=$ $11,288)$.

\begin{tabular}{|c|c|c|}
\hline Characteristics & $\mathrm{n}$ & $\%$ \\
\hline \multicolumn{3}{|l|}{ Demographics } \\
\hline Age, yrs, mean (SD) & $39.41(15.48)$ & \\
\hline Sex: male/female, $\mathrm{n}$ & $1420 / 9868$ & \\
\hline \multicolumn{3}{|l|}{ Index diagnosis yr } \\
\hline 2001-2004 & 4949 & 43.84 \\
\hline $2005-2008$ & 3622 & 32.09 \\
\hline 2009-2012 & 2717 & 24.07 \\
\hline \multicolumn{3}{|c|}{ Comorbidity, 12 mos before index date } \\
\hline Cerebrovascular disease & 535 & 4.74 \\
\hline Ischemic heart disease & 724 & 6.41 \\
\hline Diabetes mellitus & 679 & 6.02 \\
\hline Dyslipidemia & 1054 & 9.34 \\
\hline Renal dysfunction & 831 & 7.36 \\
\hline \multicolumn{3}{|c|}{ SLE severity index, 6 mos after cohort entry date } \\
\hline Mild & 4109 & 36.40 \\
\hline Moderate & 3881 & 34.38 \\
\hline Severe & 3298 & 29.22 \\
\hline \multicolumn{3}{|c|}{ SLE medication, 12 mos after cohort entry date } \\
\hline Glucocorticoid & 10,415 & 92.27 \\
\hline Injectable form & 4568 & 40.47 \\
\hline \multicolumn{3}{|l|}{ Other SLE medications } \\
\hline NSAID & 8954 & 79.32 \\
\hline Hydroxychloroquine & 8998 & 79.71 \\
\hline Azathioprine & 3534 & 31.31 \\
\hline Cyclophosphamide & 1707 & 15.12 \\
\hline Mycophenolate mofetil & 221 & 1.96 \\
\hline Methotrexate & 591 & 5.24 \\
\hline Leflunomide & 35 & 0.31 \\
\hline Chlorambucil & 22 & 0.19 \\
\hline Rituximab & 3 & 0.03 \\
\hline \multicolumn{3}{|c|}{ Average daily prednisolone-equivalent dose, $\mathrm{mg}$, first $3 \mathrm{mos}$} \\
\hline Mean (SD) & $56.73(447.66)$ & \\
\hline Median & 15.38 & \\
\hline
\end{tabular}

SLE: systemic lupus erythematosus; NSAID: nonsteroidal antiinflammatory drug.

nausea/vomiting, fatty liver, tuberculosis, fungal infection, and cardiovascular disease (Table 3 ).

\section{DISCUSSION}

To our knowledge, our study is the first to investigate the association between the longitudinal pattern of GC use (by quantifying the time-varying dose and intensity of GC) and the risk of related AE among patients with SLE using a nationwide database of ethnic Chinese. The major strength of our study is that we provide important evidence in GC-associated AE in the Asian patients with SLE, who are known to have a distinct phenotype compared to patients with SLE in Western countries. The time-varying measurement approach adopted in our study also help us to better identify the real-world disease progression, treatments, and associated AE among patients with SLE. The design of our study further permits calculation of risk ratios and a dose/exposure effect between GC used and associated
Table 2. Incidence rates of GC-related AE

\begin{tabular}{|c|c|c|c|}
\hline GC-induced AE & $\begin{array}{l}\text { Followup, } \\
\text { Yrs, mean } \\
\quad(\mathrm{SD})\end{array}$ & Events, $\mathrm{n}$ & $\begin{array}{l}\text { Incidence } \\
\text { rate/1000 } \\
\text { person-yrs }\end{array}$ \\
\hline \multicolumn{4}{|l|}{ Musculoskeletal disorders } \\
\hline Osteoporosis & $5.56(3.61)$ & 1769 & 28.17 \\
\hline Osteonecrosis & $6.11(3.59)$ & 444 & 6.44 \\
\hline Fractures & $5.77(3.60)$ & 1548 & 23.77 \\
\hline Myopathy & $6.25(3.59)$ & 79 & 1.12 \\
\hline \multicolumn{4}{|l|}{ Gastrointestinal disorders } \\
\hline Peptic ulcer & $3.75(3.32)$ & 6251 & 147.84 \\
\hline Viral hepatitis & $6.18(3.61)$ & 305 & 4.37 \\
\hline Pancreatitis & $6.22(3.59)$ & 209 & 2.98 \\
\hline Nausea and vomiting & $5.80(3.60)$ & 1517 & 23.18 \\
\hline Fatty liver & $6.21(3.59)$ & 216 & 3.08 \\
\hline \multicolumn{4}{|l|}{ Ophthalmologic disorders } \\
\hline Cataract & $5.69(3.60)$ & 1679 & 26.16 \\
\hline Glaucoma & $6.13(3.61)$ & 431 & 6.23 \\
\hline \multicolumn{4}{|l|}{ Infectious diseases } \\
\hline Tuberculosis & $6.09(3.61)$ & 540 & 7.85 \\
\hline Fungal infection & $5.38(3.63)$ & 2568 & 42.26 \\
\hline Bacterial infection & $3.56(3.81)$ & 5489 & 136.42 \\
\hline \multicolumn{4}{|l|}{ Cardiovascular disorders } \\
\hline Dyslipidemia & $5.62(3.61)$ & 1709 & 26.94 \\
\hline Atherosclerosis & $6.11(3.59)$ & 524 & 7.60 \\
\hline Ischemic heart disease & $6.06(3.59)$ & 665 & 9.72 \\
\hline Heart failure & $6.08(3.60)$ & 701 & 10.21 \\
\hline Hypertension & $5.41(3.64)$ & 2301 & 37.67 \\
\hline \multicolumn{4}{|l|}{ Neuropsychiatric disorders } \\
\hline Psychosis & $6.24(3.60)$ & 120 & 1.70 \\
\hline Depression & $5.89(3.61)$ & 1002 & 15.07 \\
\hline Cerebrovascular & $5.99(3.60)$ & 940 & 13.90 \\
\hline Insomnia & $4.23(3.57)$ & 4019 & 78.64 \\
\hline \multicolumn{4}{|l|}{ Metabolic/endocrine disorders } \\
\hline Obesity & $6.26(3.59)$ & 80 & 1.13 \\
\hline Gout and hyperuricemia & $5.55(3.62)$ & 1868 & 29.81 \\
\hline Cushingoid syndrome & $6.22(3.59)$ & 184 & 2.62 \\
\hline Acne vulgaris & $5.67(3.65)$ & 1447 & 22.62 \\
\hline
\end{tabular}

GC: glucocorticoid; AE: adverse event.

AE. Overall, we found that both dose and intensity of GC use were associated with $\mathrm{AE}$.

For some specific GC-associated $\mathrm{AE}$ such as osteonecrosis, osteoporosis, bacterial infection, Cushingoid syndrome, and sleep disorder, a joint effect of dose and intensity was observed. Patients who received both high-dose and high-intensity GC treatment had the highest risk of these AE. However, even those who received both low-dose and low-intensity GC treatment had a higher risk of these $\mathrm{AE}$ compared with patients who did not receive GC. These results agree with previous studies suggesting that no consensus about a "safe" dose can be reached because even low-dose GC may cause the loss of bone mineral density (BMD) and Cushingoid syndrome $e^{23,24}$.

$\mathrm{GC}$-induced osteoporosis is one of the most common AE associated with $\mathrm{GC}$ use. We found that patients who received both a high dose and a high intensity of GC had the highest risk of osteoporosis followed by those who received a low 
Table 3. Association between intensity and dose of GC use and GC-associated AE. Values are aOR* (95\% CI) unless otherwise specified.

Variables

\begin{tabular}{|c|c|c|c|c|}
\hline \multicolumn{5}{|l|}{ Category } \\
\hline $\begin{array}{l}\text { Average prednisolone-equivalent } \\
\text { dose, } \mathrm{mg} / \text { day }\end{array}$ & $\leq 10 \mathrm{mg} /$ day $($ low-dose $)$ & $\leq 10 \mathrm{mg} /$ day (low-dose) & $>10 \mathrm{mg} /$ day (high-dose) & $>10 \mathrm{mg} /$ day (high-dose) \\
\hline Medication possession ratio & $<80 \%$ (low-intensity) & $\geq 80 \%$ (high-intensity) & $<80 \%$ (low-intensity) & $\geq 80 \%$ (high-intensity) \\
\hline \multicolumn{5}{|l|}{ Musculoskeletal disorders } \\
\hline Osteoporosis & $1.71(1.23-2.37)$ & $2.19(1.62-2.97)$ & $1.55(1.10-2.20)$ & $3.67(2.60-5.18)$ \\
\hline Osteonecrosis & $2.87(1.16-7.08)$ & $5.04(2.14-11.91)$ & $4.02(1.70-9.49)$ & $9.09(3.70-22.33)$ \\
\hline Fracture & $1.01(0.72-1.41)$ & $1.17(0.86-1.61)$ & $1.53(1.12-2.08)$ & $1.35(0.88-2.05)$ \\
\hline Myopathy & $0.91(0.08-10.31)$ & $0.71(0.05-9.98)$ & $8.92(1.72-46.18)$ & $12.43(2.31-66.98)$ \\
\hline \multicolumn{5}{|l|}{ Gastrointestinal disorders } \\
\hline Peptic ulcer & $1.17(0.98-1.39)$ & $1.26(1.06-1.49)$ & $2.14(1.83-2.50)$ & $2.36(1.95-2.86)$ \\
\hline Viral hepatitis & $1.23(0.57-2.63)$ & $2.34(1.24-4.43)$ & $2.35(1.23-4.49)$ & $3.82(1.94-7.53)$ \\
\hline Pancreatitis & $0.93(0.31-2.80)$ & $0.76(0.25-2.28)$ & $2.26(0.96-5.34)$ & $2.27(0.80-6.49)$ \\
\hline Nausea and vomiting & $1.05(0.77-1.43)$ & $1.26(0.93-1.70)$ & $1.52(1.12-2.06)$ & $1.61(1.13-2.30)$ \\
\hline Fatty liver & $1.62(0.71-3.68)$ & $0.70(0.26-1.91)$ & $3.04(1.47-6.27)$ & $2.97(1.28-6.92)$ \\
\hline \multicolumn{5}{|l|}{ Ophthalmologic disorders } \\
\hline Cataracts & $1.28(0.92-1.77)$ & $1.74(1.29-2.34)$ & $1.68(1.22-2.31)$ & $2.63(1.86-3.74)$ \\
\hline Glaucoma & $1.30(0.61-2.74)$ & $1.41(0.66-2.98)$ & $2.72(1.37-5.37)$ & $5.88(2.94-11.78)$ \\
\hline \multicolumn{5}{|l|}{ Infectious diseases } \\
\hline Tuberculosis & $1.14(0.63-2.06)$ & $0.64(0.32-1.27)$ & $2.35(1.43-3.87)$ & $4.39(2.50-7.70)$ \\
\hline Fungal infection & $1.28(0.97-1.67)$ & $1.31(1.00-1.72)$ & $2.49(1.96-3.16)$ & $3.84(2.93-5.03)$ \\
\hline Bacterial infection & $2.12(1.73-2.60)$ & $2.19(1.78-2.69)$ & $3.26(2.69-3.94)$ & $3.89(3.09-4.89)$ \\
\hline \multicolumn{5}{|l|}{ Neuropsychiatric disorders } \\
\hline Psychosis & $1.21(0.33-4.52)$ & $1.08(0.24-4.87)$ & $2.14(0.72-6.37)$ & $3.44(0.91-12.93)$ \\
\hline Depression & $1.69(1.19-2.39)$ & $1.12(0.76-1.65)$ & $1.50(1.02-2.20)$ & $2.77(1.86-4.13)$ \\
\hline Sleep disorder & $1.42(1.15-1.74)$ & $1.32(1.07-1.64)$ & $2.19(1.80-2.66)$ & $3.59(2.89-4.47)$ \\
\hline \multicolumn{5}{|l|}{ Cardiovascular disorders } \\
\hline Dyslipidemia & $0.88(0.64-1.23)$ & $1.12(0.83-1.51)$ & $1.38(1.02-1.85)$ & $2.44(1.79-3.34)$ \\
\hline Cerebrovascular disease & $2.10(1.37-3.22)$ & $1.48(0.93-2.34)$ & $3.36(2.26-5.00)$ & $5.48(3.46-8.65)$ \\
\hline Hypertension & $1.19(0.87-1.63)$ & $1.28(0.95-1.72)$ & $2.44(1.86-3.20)$ & $4.30(3.24-5.70)$ \\
\hline Atherosclerosis & $1.34(0.79-2.28)$ & $0.91(0.52-1.61)$ & $1.56(0.96-2.54)$ & $2.02(1.10-3.70)$ \\
\hline Ischemic heart disease & $0.97(0.59-1.61)$ & $0.87(0.54-1.39)$ & $1.74(1.15-2.64)$ & $2.09(1.26-3.48)$ \\
\hline Heart failure & $1.67(0.94-2.98)$ & $1.14(0.62-2.08)$ & $4.39(2.78-6.91)$ & $6.40(3.79-10.83)$ \\
\hline \multicolumn{5}{|l|}{ Metabolic/endocrine disorders } \\
\hline Obesity & $0.63(0.16-2.47)$ & $0.28(0.06-1.41)$ & $1.26(0.41-3.89)$ & $1.13(0.29-4.39)$ \\
\hline Gout and hyperuricemia & $1.33(0.95-1.86)$ & $1.50(1.09-2.06)$ & $2.18(1.61-2.95)$ & $3.56(2.54-4.99)$ \\
\hline Cushingoid syndrome & $6.51(1.19-35.62)$ & $10.03(1.95-51.57)$ & $18.21(3.79-87.56)$ & $62.03(12.59-305.69)$ \\
\hline Acne vulgaris & $1.36(1.00-1.86)$ & $1.63(1.22-2.18)$ & $1.82(1.33-2.48)$ & $2.04(1.44-2.89)$ \\
\hline
\end{tabular}

*Adjusted for patient demographics (age, sex), comorbidities, concomitant SLE treatment, and a propensity score to address the potential selection bias among patients receiving high doses of GC. GC: glucocorticoid; AE: adverse event; aOR: adjusted OR; SLE: systemic lupus erythematosus.

dose and a high intensity of GC. This may reveal that high-intensity GC use is more influential than average GC daily dose on the increased risk of osteoporosis. This is also in line with previous studies suggesting that although there is a strong correlation between cumulative dose and loss of $\mathrm{BMD}$, discontinuation of these drugs may interrupt their damage to bone tissue ${ }^{10,25}$. However, we found that a high daily dose of GC resulted in a statistically significant increased risk for osteoporosis, even administered at low intensity, as has been suggested in previous studies $9,26,27$.

Our finding that the likelihood of developing certain GC-related AE (including myopathy, glaucoma, nausea/ vomiting, fatty liver, tuberculosis, fungal infection, and cardiovascular disease) was associated with high-dose GC therapy is also consistent with previous studies ${ }^{8,26}$. Our study showed that the risk of bacterial infection increased with the GC daily dose ${ }^{28,29,30}$. In addition, our findings supported previous results that opportunistic infections s, $^{7,31}$ such as tuberculosis and fungal infections were associated with GC use in a dose-response relationship ${ }^{32}$. An explanation for such results, suggested previously, is that suppression of tuberculin reactivity results when a prednisolone-equivalent dose $>15$ $\mathrm{mg}$ /day is administered for 2-4 weeks ${ }^{33}$. In addition, our study demonstrated increased risk of viral infection associated with GC use, except in the low-intensity and low-dose GC group. A previous study has suggested, counter to our finding, that even a minimal level of GC use can stimulate the reactivation of latent viruses and lead to clinical infection among patients with SLE ${ }^{31}$.

Again, high-intensity GC use played an important role in Personal non-commercial use only. The Journal of Rheumatology Copyright @ 2018 . All rights reserved. 
the GC-associated ophthalmologic AE considered in our study. For example, our findings are consistent with previous studies that steroid-induced glaucoma is attributable to persistent systemic GC therapy ${ }^{34,35}$. Our findings also showed that low-dose, high-intensity GC treatment was associated with increased risk of cataracts, which is in line with previous findings that increased risk of cataracts was associated with cumulative exposure to GC, even under low-dose GC treatment ${ }^{10,31}$. Similar results were also found regarding peptic ulcer ${ }^{36}$, acne vulgaris, and hyperuricemia.

In our study, no association was found between GC use and the occurrence of some of the AE considered, such as pancreatitis, psychosis, and obesity. A likely explanation for this is that some AE may be associated with GC use as well as with the progression of SLE disease $e^{4,10,11,37}$. For example, it is very difficult to evaluate the effect of GC use on the risk of developing neuropsychiatric $\mathrm{AE}$, such as depression, without confounding by SLE. It is well known that depression is the most common psychiatric manifestation in $\mathrm{SLE}^{38,39}$. On the other hand, sleep disorders appear to be associated with psychosocial factors due to disease severity of SLE (e.g., depression, fatigue, and pain) and steroid therapy 38,40 .

Although our study provides valuable insights into the association between longitudinal patterns of GC use and the risk of GC-related AE, as with all observational studies based on claims databases, it has limitations. First, using the ICD-9-CM code to identify the study endpoints may underestimate the incidence of GC-related AE. However, we also included drugs used to treat $\mathrm{GC}$-related $\mathrm{AE}$ to improve the validity of the estimates. In addition, the $\mathrm{AE}$ may be underreported in our retrospective study as compared to a more prospective cohort study. Second, we were unable to collect data on variables not routinely identified in claims databases, such as patient disease severity and laboratory data. However, we used the SLE severity index to categorize a patient's disease severity $^{22}$, and we adopted the propensity score technique to adjust for the baseline differences among patients with SLE. Third, the incidence rates in our study may be different from those in other studies. This may be explained by geographic and racial differences in patients with SLE worldwide ${ }^{41}$. Fourth, while our study retrieved all parenteral and oral systemic GC for our study cohort to help us better identify the real-world disease progression, treatments, and associated $\mathrm{AE}$ among the patients with SLE, methylprednisolone pulses could activate a different pathway of AE. Fifth, the cutoff of a 10-mg prednisolone-equivalent daily dose was different from that used in other studies in Western countries. For example, Ruiz-Arruza, et al used $7.5 \mathrm{mg} /$ day as the cutoff $^{8}$. We used $10 \mathrm{mg} /$ day as the cutoff to reflect the clinical practice in Taiwan. In Taiwan, the smallest dosage of steroids is $5 \mathrm{mg} / \mathrm{tablet}$ and clinicians here usually prescribe 2 pills $(10 \mathrm{mg})$ for someone who may need a medium-high dose of steroids (i.e., $7.5 \mathrm{mg} /$ day). We also have retrieved our data and found that very few patients in Taiwan use $7.5 \mathrm{mg} / \mathrm{day}$ of steroids. We therefore decided to use $10 \mathrm{mg} /$ day as the cutoff.

Our empirical analysis found that dose and intensity of GC use were both associated with higher risk of AE. To our knowledge, ours is the first study to document the association between longitudinal use of GC and risk of $\mathrm{AE}$ among a nationwide Asian SLE cohort.

\section{ACKNOWLEDGMENT}

The authors acknowledge the National Health Insurance Administration and National Health Research Institutes for making available the databases for this study.

\section{ONLINE SUPPLEMENT}

Supplementary material accompanies the online version of this article.

\section{REFERENCES}

1. Petri M. Epidemiology of systemic lupus erythematosus. Best Pract Res Clin Rheumatol 2002;16:847-58.

2. Mak A, Cheung MW, Chiew HJ, Liu Y, Ho RC. Global trend of survival and damage of systemic lupus erythematosus: meta-analysis and meta-regression of observational studies from the 1950s to 2000s. Semin Arthritis Rheum 2012;41:830-9.

3. Doria A, Gatto M, Zen M, Iaccarino L, Punzi L. Optimizing outcome in SLE: treating-to-target and definition of treatment goals. Autoimmun Rev 2014;13:770-7.

4. Chambers SA, Allen E, Rahman A, Isenberg D. Damage and mortality in a group of British patients with systemic lupus erythematosus followed up for over 10 years. Rheumatology 2009;48:673-5.

5. Al-Maini M, Urowitz M. Systemic steroids. In: Tsokos GC, Gordon C, Smolen JS, editors. Systemic lupus erythematosus: a companion to rheumatology. Philadelphia: Moseby Elsevier; 2007:487-97.

6. Thamer M, Hernán MA, Zhang Y, Cotter D, Petri M. Prednisone, lupus activity, and permanent organ damage. J Rheumatol 2009;36:560-4

7. Shah M, Chaudhari S, McLaughlin TP, Kan HJ, Bechtel B, Dennis GJ, et al. Cumulative burden of oral corticosteroid adverse effects and the economic implications of corticosteroid use in patients with systemic lupus erythematosus. Clin Ther 2013;35:486-97.

8. Ruiz-Arruza I, Ugarte A, Cabezas-Rodriguez I, Medina JA, Moran MA, Ruiz-Irastorza G. Glucocorticoids and irreversible damage in patients with systemic lupus erythematosus. Rheumatology 2014;53:1470-6.

9. Petri M, Bechtel B, Dennis G, Shah M, McLaughlin T, Kan H, et al. Burden of corticosteroid use in patients with systemic lupus erythematosus: results from a Delphi panel. Lupus 2014:23:1006-13.

10. Zonana-Nacach A, Barr SG, Magder LS, Petri M. Damage in systemic lupus erythematosus and its association with corticosteroids. Arthritis Rheum 2000;43:1801-8

11. Gladman DD, Urowitz MB, Rahman P, Ibañez D, Tam LS. Accrual of organ damage over time in patients with systemic lupus erythematosus. J Rheumatol 2003;30:1955-9.

12. Sarnes E, Crofford L, Watson M, Dennis G, Kan H, Bass D. Incidence and US costs of corticosteroid-associated adverse events: a systematic literature review. Clin Ther 2011;33:1413-32.

13. Ruiz-Irastorza G, Danza A, Khamashta M. Glucocorticoid use and abuse in SLE. Rheumatology 2012;51:1145-53.

14. Zahr ZA, Fang H, Magder LS, Petri M. Predictors of corticosteroid tapering in SLE patients: the Hopkins Lupus Cohort. Lupus 2013;22:697-701.

Personal non-commercial use only. The Journal of Rheumatology Copyright (C) 2018. All rights reserved 
15. Bertsias GK, Ioannidis JP, Boletis J, Bombardieri S, Cervera R, Dostal C, et al; Task Force of the EULAR Standing Committee for International Clinical Studies Including Therapeutics. EULAR recommendations for the management of systemic lupus erythematosus. Report of a Task Force of the EULAR Standing Committee for International Clinical Studies Including Therapeutics. Ann Rheum Dis 2008;67:195-205.

16. Hsiao FY, Yang CL, Huang YT, Huang WF. Using Taiwan's National Health Insurance research databases for pharmacoepidemiology research. J Food Drug Anal 2007;15:99-108.

17. Moores KG, Sathe NA. A systematic review of validated methods for identifying systemic lupus erythematosus (SLE) using administrative or claims data. Vaccine 2013;31 Suppl 10:K62-73.

18. Chang JS, Tsai CR, Tsai YW, Wiemels JL. Medically diagnosed infections and risk of childhood leukaemia: a population-based case-control study. Int J Epidemiol 2012;41:1050-9.

19. Chen YM, Chen DY, Chen LK, Tsai YW, Chang LC, Huang WF, et al. Alendronate and risk of esophageal cancer: a nationwide population-based study in Taiwan. J Am Geriatr Soc 2011; 59:2379-81

20. Hess LM, Raebel MA, Conner DA, Malone DC. Measurement of adherence in pharmacy administrative databases: a proposal for standard definitions and preferred measures. Ann Pharmacother 2006;40:1280-8.

21. Austin PC. An introduction to propensity score methods for reducing the effects of confounding in observational studies. Multivariate Behav Res 2011;46:399-424.

22. Garris CP, Jhingran PM, Engel-Nitz NM, Riedel AA, Goldberg G, Bass DL, et al. Assessing systemic lupus erythematosus disease severity and disease flares: development of a claims-based algorithm [abstract]. Arthritis Rheum 2010;62 Suppl 10:757.

23. Da Silva JA, Jacobs JW, Kirwan JR, Boers M, Saag KG, Inês LB, et al. Safety of low dose glucocorticoid treatment in rheumatoid arthritis: published evidence and prospective trial data. Ann Rheum Dis 2006;65:285-93.

24. Saag KG, Furst DE. Major side effects of systemic glucocorticoids. [Internet. Accessed September 12, 2017.] Available from: www.uptodate.com/contents/major-side-effects-of-systemicglucocorticoids

25. Vestergaard P. Skeletal effects of systemic and topical corticosteroids. Curr Drug Saf 2008;3:190-3.

26. van Staa TP, Leufkens HG, Cooper C. The epidemiology of corticosteroid-induced osteoporosis: a meta-analysis. Osteoporos Int 2002; 13:777-87

27. van Staa TP, Leufkens HG, Abenhaim L, Zhang B, Cooper C. Oral corticosteroids and fracture risk: relationship to daily and cumulative doses. Rheumatology 2000;39:1383-9.
28. Migita K, Sasaki Y, Ishizuka N, Arai T, Kiyokawa T, Suematsu E, et al. Glucocorticoid therapy and the risk of infection in patients with newly diagnosed autoimmune disease. Medicine 2013;92:285-93.

29. Dixon WG, Kezouh A, Bernatsky S, Suissa S. The influence of systemic glucocorticoid therapy upon the risk of non-serious infection in older patients with rheumatoid arthritis: a nested case-control study. Ann Rheum Dis 2011;70:956-60.

30. McDonough AK, Curtis JR, Saag KG. The epidemiology of glucocorticoid-associated adverse events. Curr Opin Rheumatol 2008;20:131-7.

31. Fardet L, Kassar A, Cabane J, Flahault A. Corticosteroid-induced adverse events in adults: frequency, screening and prevention. Drug Saf 2007;30:861-81.

32. Jick SS, Lieberman ES, Rahman MU, Choi HK. Glucocorticoid use, other associated factors, and the risk of tuberculosis. Arthritis Rheum 2006;55:19-26.

33. American Thoracic Society. Targeted tuberculin testing and treatment of latent tuberculosis infection. MMWR Recomm Rep 2000;49:1-51

34. Jones R 3rd, Rhee DJ. Corticosteroid-induced ocular hypertension and glaucoma: a brief review and update of the literature. Curr Opin Ophthalmol 2006;17:163-7.

35. Tripathi RC, Parapuram SK, Tripathi BJ, Zhong Y, Chalam KV. Corticosteroids and glaucoma risk. Drugs Aging 1999;15:439-50

36. García Rodríguez LA, Hernández-Díaz S. The risk of upper gastrointestinal complications associated with nonsteroidal anti-inflammatory drugs, glucocorticoids, acetaminophen, and combinations of these agents. Arthritis Res 2001;3:98-101.

37. Urowitz MB, Gladman DD, Ibañez D, Fortin PR, Bae SC, Gordon $\mathrm{C}$, et al. Evolution of disease burden over five years in a multicenter inception systemic lupus erythematosus cohort. Arthritis Care Res 2012;64:132-7.

38. Palagini L, Tani C, Mauri M, Carli L, Vagnani S, Bombardieri S, et al. Sleep disorders and systemic lupus erythematosus. Lupus 2014;23:115-23.

39. Meszaros ZS, Perl A, Faraone SV. Psychiatric symptoms in systemic lupus erythematosus: a systematic review. J Clin Psychiatry 2012;73:993-1001.

40. Da Costa D, Bernatsky S, Dritsa M, Clarke AE, Dasgupta K, Keshani A, et al. Determinants of sleep quality in women with systemic lupus erythematosus. Arthritis Rheum 2005;53:272-8.

41. Danchenko N, Satia JA, Anthony MS. Epidemiology of systemic lupus erythematosus: a comparison of worldwide disease burden. Lupus 2006;15:308-18. 